\title{
Curación y almacenaje de fuentes de información académica, profesional e laboral para el módulo de FOL
}

\author{
César Pérez Rama \\ Facultad de Ciencias de la Educación. Universidad de La Coruña
}

\begin{abstract}
Resumen
En el campo de la orientación profesional la red ofrece múltiples posibilidades de utilización: como fuente de información académica, profesional y laboral, como medio para la propia formación, como recurso didáctico y como medio para vehicular algunas de las funciones orientadoras.

La cantidad ingente de recursos existentes en la red hacen imprescindible un trabajo previo de curación y difusión de los mismos de forma que se facilite el trabajo de los profesionales de la orientación. En la comunicación presentamos un catálogo de fuentes de información académica, profesional y laboral para el profesorado de Formación y orientación laboral (FOL) y para el alumnado de los ciclos formativos. La idea del catálogo y el procedimiento de elaboración se inspiran en un trabajo previo de características semejantes para el profesorado tutor y para las orientadoras y orientadores de la ESO (Arza y Reimundez, 2014).
\end{abstract}

Palabras clave: orientación profesional, información profesional, áreas de intervención, módulo de FOL, formación profesional, web 2.0.

\section{Conceptualización de la OP}

La orientación profesional constituye un elemento clave en la vida de las personas. La principal característica conceptual que se le atribuye a la orientación se deriva de su concepción más contemporánea como un proceso continuo que acompaña a lo largo de la vida de las personas en la toma de decisiones de formación y empleo. También se le atribuyen otra serie de características clave que la completan en su visión teórica, a saber: preventiva, continua, formativa y para la transformación y el cambio (Arza, 2002).

En cuanto a los modelos de intervención que la definen en la práctica, debemos decir que existe un consenso a la hora de determinar tres modelos básicos de intervención en la orientación profesional: el clínico o de consejo, el de consulta o asesoramiento y el de programas (Álvarez \& Isus, 1998; Álvarez, 2009; Álvarez \& Bisquerra, 2012). Estos se identifican en función de tres modelos básicos en función de tres características: el tipo de intervención, que puede ser individual o grupal; la finalidad (preventiva o remedial) y el tipo de intervención (directa o indirecta). A pesar de ello, autores como Álvarez González (1998), Benavent (1999) o Pantoja y Campoy (2001), citados en Ceinos Sanz, 2009 proponen un cuarto modelo, el tecnológico, entendiendo por modelo tecnológico aquel que trata de canalizar la acción orientadora a través, exclusivamente, de la ayuda de las nuevas herramientas tecnológicas (como pueden ser los medios telemáticos) donde no existe un contacto físico directo entre orientador/a y orientado/a.

En este sentido coincidimos con autores como Malik (1998), Repetto (2002) o Santana (2003) citados en Ceinos Sanz, 2009 en que el uso de las nuevas herramientas tecnológicas no podrán constituir por si solas un modelo de intervención único e independiente, sino que se deberá de concebir como un instrumento de apoyo que facilite, y en complementación con los restantes modelos, la práctica orientadora. Si hay un modelo que se ha de potenciar es el modelo de programas, porque es el que, según Álvarez González (2009) mejor asume los principios y funciones de la OP y el que implica a un mayor número de agentes, aun siendo el más exigente en su realización.

Otra de las dimensiones que completan la conceptualización de la OP es la delimitación de sus áreas de intervención, que aluden a aquellos elementos o contenidos que ésta debe de abordar, fundamentalmente, a la hora de llevar a cabo actividades educativas pues estas nos ayudarán a delimitar los contenidos objeto de estudio, como ocurre por ejemplo en el módulo de FOL. Existen distintas propuestas de áreas (entre otras, Sánchez García, 2004, 2013; Romero, 2002; Álvarez González e Isus, Álvarez González, 1995).

Aunque todavía no existe un consenso totalmente claro acerca de las áreas de la orientación profesional, en la mayoría de las propuestas encontramos, fundamentalmente, un denominador común que se concretiza en cuatro áreas, a saber: el área del autoconocimiento o del conocimiento de sí mismo, el conocimiento del sistema educativo y del mundo laboral y la toma de decisiones. La información como se puede ver está vinculada a la información académica, profesional y laboral. En la parte informativa de la orientación profesional es en la que vamos a centrar la curación de contenidos, pero centrada en las necesidades del alumnado que curso formación profesional inicial.

\section{La orientación profesional en el módulo de FOL}

El módulo de FOL es un módulo transversal que se imparte en todos los ciclos formativos de grado medio y superior, y que constituye una herramienta imprescindible para la transición de la escuela a la vida activa. El módulo abarca cuatro grandes bloques 
repartidos en dos unidades formativas: "prevención de riesgos laborales" y "equipos de trabajo, derecho del trabajo y de la seguridad social y búsqueda de empleo" (unidad formativa dos). En la última unidad formativa están presentes contenidos de orientación profesional. En el curriculum de FOL recobran una especial importancia la dimensión informativa de la orientación profesional, especialmente la información sobre las opciones de formación y sobre el mercado laboral. Además de estos contenidos aparecen los relativos al autoconocimiento y a las técnicas y estrategias de búsqueda de empleo. A pesar de que ciertos estudios el ámbito de la orientación profesional (Sanjuán, 2010), donde la OP ha sido uno de los ámbitos más valorados por los profesores de FOL, ésta no posee todo el peso que debería en el curriculum oficial de FOL. Algunas de estas deficiencias hacen alusión a la falta de la inclusión del enfoque de género, del manejo de las TIC en el proceso de búsqueda de empleo y la presentación de los contenidos como un simple listado sin ningún tipo de organización y estructura.

\section{Los ámbitos de la utilización de internet en la OP}

La web 2.0 nos ofrece multitud de herramientas de gran utilidad en el campo de la orientación profesional, entre las que podemos destacar los blogs, las webquest, las wikis, los marcadores sociales, las comunidades (como youtube, wikipedia Facebook, twitter...) y que por lo tanto pueden ser susceptibles de utilización por parte de los profesionales de la orientación para llevar a cabo su labor. En esta línea las posibilidades de utilización en los que podríamos agrupar las posibilidades o utilidades que internet nos ofrece serían (Arza 2015, Baelo, 2013): herramienta para la búsqueda de empleo, recurso para la formación y el desarrollo profesional, fuente de información académica y profesional, contenido y objeto de la intervención, recurso para vehicular algunas de las funciones orientadoras.

La selección de recursos que presentamos se vincula con uno de los ámbitos de utilización descritos, como fuente de información académica, profesional y laboral. En la web se encuentran multitud de recursos que nos proporcionan este tipo de información y que el profesorado de FOL deberá de conocer y seleccionar en función de las necesidades informativas del alumnado. La información académica, profesional y laboral es fundamental para que el alumnado pueda construir su itinerario de formación y acceder al mercado laboral.

\section{Catálogo de recursos de información académica profesional y laboral}

Esta parte del catálogo forma parte de uno más amplio que quiere abarcar los distintos ámbitos de utilización de la red enumerados con anterioridad. En cuanto al procedimiento de confección del mismo, han sido 4 los pasos que se han llevado a cabo y que se corresponden con el proceso de curación de contenidos. Estos han sido: localización, criterios de selección, clasificación y análisis y almacenaje.

\subsection{Localización}

En la fase de búsqueda se ha realizado un gran esfuerzo para realizar una selección de recursos útiles para el desarrollo de las funciones informativas del profesorado de la especialidad de FOL en el ámbito de la orientación profesional, y especialmente en el módulo de FOL. Google, google académico, dialnet y plataformas como educaweb y edublog, fueron los principales focos de localización de los recursos. Estas plataformas de búsqueda nos condujeron a otros recursos interesantes por el hecho de que éstos ya se encontraban previamente seleccionados.

\subsection{Criterios de selección}

En cuanto a los criterios de selección utilizados han sido fundamentalmente:

- La utilidad valorada en función del tipo de información que necesita el profesorado de FOL y el alumnado para la creación de un itinerario de formación y para la búsqueda de empleo. En coherencia con este criterio los recursos seleccionados sirven para varias etapas educativas, formación profesional y educación secundaria

- Autoría de la creación y gestión del recurso. Este criterio se aplicó, fundamentalmente para la información legal, la información estadística sobre el mercado laboral y la información sobre formación y empleo. En estos casos, se seleccionaron los recursos procedentes de organismos e entidades públicas, empresas de recursos humanos y portales de empleo de amplia tradición.

- Los premios recibidos y la consideración de buenas prácticas fundamentalmente en la selección de recursos en los que se ha apostado por la inclusión de las tecnologías de la información y la comunicación.

- La actualización de la información, funcionamiento de los enlaces propuestos o número de visitas fueron otros de los criterios utilizados.

- Usabilidad o facilidad en el uso del recurso. Este criterio es especialmente importante, no nos debemos de olvidar de su manejo fácil e intuitivo. Una buena interfaz y un contenido interactivo lo convierten en un recurso atractivo y motivador para el alumnado.

\subsection{Categorías de clasificación}

La delimitación de las categorías de clasificación es fundamental para una buena organización de los recursos, para su posterior almacenaje y para facilitar su recuperación. Cada recurso se clasifica con varias categorías. Las categorías que utilizamos son de cuatro tipos:

a) Las relativas al soporte del recurso: documento, vídeo, web, blog, wiki

b) Las relacionadas con las personas destinatarias del recurso: alumnado, profesorado tutor, profesorado de FOL, orientadores/as, todos. 
c) Las relacionadas con la etapa educativa: ESO, Formación Profesional, Bachillerato y todas.

d) Las relacionadas con los contenidos de la información: información académica (referida a las oportunidades de formación y a las becas y ayudas para el estudio) información profesional (referida al perfil profesional: competencias profesional, funciones etc.) e información laboral (datos estadísticos de empleo, fuentes de información sobre empleo, portales de empleo etc.). La mayor parte de los recursos seleccionados se circunscribe a webs de carácter institucional, que nos proporcionan información sobre las opciones de formación en el sistema educativo y laboral. Su gestión se lleva a cabo, fundamentalmente, por organismos públicos, tanto a nivel estatal como autonómico, con competencias en el ámbito educativo y laboral.

\subsection{Análisis y almacenaje}

Para realizar el análisis de los recursos seleccionados se creó una ficha en la cual se recogía la siguiente información: datos de identificación (nombre, autor/autores y la dirección web del recurso), categoría de la clasificación, descripción, en la que se resumen los contenidos y finalmente una valoración, principalmente, en relación con su utilidad y con las temáticas de la orientación profesional que aborda.

Para el almacenaje de los recursos utilizamos el marcador social "Diigo". Esta herramienta de la web 2.0 nos permite, almacenar, recuperar y compartir recursos de todo tipo.

Una de las desventajas de los marcadores sociales es la clasificación de los recursos, ya que cada usuario utiliza las que considera convenientes. Para tratar de minimizar este problema, a partir de las categorías descritas con anterioridad hemos desarrollado subcategorías que nos van a permitir una mejor clasificación y recuperación de los recursos. La categorización de los recursos en el marcador social ha seguido los mismos parámetros que los que exponemos aquí, si bien esta plataforma nos ha permitido asignarle más de una categoría a aquellos recursos que abarquen varias temáticas. De esta manera se ha conseguido, por un lado, mejorar la búsqueda de dicho recurso y por otro hacer que su categorización fuese más completa y versátil. Diigo es una plataforma de sencillo uso y se divide, fundamentalmente, en cuatro apartados: "My library", donde encontramos los marcadores o favoritos que iremos guardando en la nube, "My network", donde encontraremos los MM.SS de las personas a las que seguimos, "My groups", donde podemos compartir determinados favoritos o marcadores con determinados grupos creados por nosotros o a los que nos hemos adherido, y por último "Comunity", donde podemos ver los marcadores que en cada momento se están subiendo y compartiendo en la red social (para aquellos asiduos al twitter sería como el “trending topic" del mismo.

\subsection{Recursos seleccionados}

En este apartado presentaremos parte de los recursos que hemos seleccionado de la red referentes a los contenidos de información académica, profesional y laboral dentro del ámbito de la orientación profesional y del módulo de FOL. En este sentido hemos subcategorizado en tres apartados estos recursos, a saber: información académica, información acerca del mercado laboral, información sobre autoempleo e información sobre empleo público.

\subsubsection{Información académica}

Dentro de este apartado acogemos algunas de las páginas web que ofrecen información académico profesional de interés para los estudiantes, fundamentalmente, de información profesional.

a) Xunta de Galicia - FP. Web autonómica que ofrece una completa información acerca de la formación profesional en Galicia. Se encuentra dividida en 8 apartados: “conoce la FP" donde nos informa sobre los requisitos y pasos básicos para acceder a la FP en nuestra comunidad autónoma y resuelve las dudas más frecuentes y las salidas académicas y profesionales que brindan los distintos ciclos. "Ven a la FP", donde nos proporciona información sobre los criterios de admisión y las distintas convocatorias de ciclos, “acreditación”, en la que nos informa ampliamente sobre las distintas posibilidades de acreditaciones de las competencias profesionales, "Probas”, información sobre las diferentes pruebas libres convocadas para el acceso a los ciclos, "validaciones", sobre equivalencias con diferentes títulos, "curriculums", donde tenemos toda la oferta formativa de los diferentes ciclos vigentes, "Normativa”, nos remite a diferentes documentos en los que se regula la FP en Galicia, y por último el apartado “empresa” en el que se hace referencia a los diferentes convenios que la comunidad autónoma posee con las empresas para la realización de los FCT y su regulación.

b) Portal Todo FP. Proporciona información sobre los estudios de formación profesional en el ámbito estatal. Se encuentra dividido en cuatro apartados: "orientación profesional”, donde podemos encontrar información sobre distintos itinerarios formativos y practicar el autoconocimiento y diferentes técnicas de búsqueda de empleo e información acerca del mercado laboral, "sobre FP”, donde encontramos información acerca de la formación profesional, las diferentes modalidades, accesos,,,etc, “qué, cómo y dónde estudiar”, a través del cual encontramos información detallada a las diferentes modalidades y opciones que nos ofrece la FP, y por último "Pruebas y convalidaciones", donde encontramos información específica en relación a las diferentes modalidades de pruebas para el acceso a la formación profesional (ciclos de grado medio, superior, para mayores de 25, y las convalidaciones, homologaciones y acreditaciones de los diferentes estudios de la formación profesional. Además también ofrece noticias actualizadas y gran cantidad de normativa referente a la formación profesional en nuestro país y en el extranjero. 
c) Ministerio de Educación, Cultura y Deporte. Becas, ayudas, subvenciones y premios que el Gobierno ofrece desde su propia página web para estudiar, para profesores, investigadores, entidades o centros docentes, de formación, para la movilidad o con el objetivo de realizar actividades extraescolares.

Además, tras registrarnos podemos activar alertas para que nos informen de las posibles becas a las que tanto alumnos como profesores podemos aspirar. Nos permite consultarlas y realizar los trámites online. Muy útil para estar al día de las becas, ayudas y subvenciones del estado.

d) Guía de nuevas profesiones. La web nos ofrece un diccionario-guía de nuevas ocupaciones organizadas por sectores. Son ocupaciones emergentes provocadas por diversas causas, nuevas demandas del mercado laboral, evolución de ocupaciones tradicionales, etc. En total se presenta información de 200 ocupaciones con una ficha de información por ocupación, que abarca los siguientes contenidos: descripción, tareas, vías de acceso, formación complementaria y tendencias del mercado.

Además se realiza una identificación y descripción del sector al que pertenece cada nueva ocupación y cada vez que entramos nos sugiere la llamada "profesión del día". Disponible en formato multimedia y texto.

e) Webs de universidades. Páginas web de las principales universidades de Galicia (Universidad de la Coruña, Universidad de Santiago, Universidad de Vigo) también proporcionan multitud de información, a través de la cual el alumnado de fases educativas terminales, se puede informar acerca de una más de las posibilidades formativas que se ofrecen.

f) Workinside. Plataforma de vídeo, que asesora en el ámbito académico y laboral mediante entrevistas audiovisuales de corta duración. Sus creadores (Anabel Jiménez, graduada en periodismo, Álvaro Checa y Pedro Fernández, licenciados en ADE y derecho) facilitan en estas micro-entrevistas el punto de vista de estudiantes universitarios, opositores y profesionales. Esta herramienta va dirigida, fundamentalmente, a alumnos de bachillerato y FP que necesitan tener más información sobre las diferentes salidas académicas y laborales. Una de sus finalidades pretende reducir el abandono universitario.

\subsubsection{Información acerca del mercado laboral}

a) Servicio público de empleo estatal (SEPE). Web muy enfocada al ámbito laboral. En ella podemos encontrar noticias e información de actualidad referentes a la situación del mercado de trabajo, estadísticas periódicas sobre empleo, contratos, prestaciones por desempleo, técnicas de búsqueda de trabajo, información para emprendedores y autónomos.... También encontramos una completa normativa referente en materia de trabajo y empleo como material de consulta actualizado (referente a protección por desempleo, formación para el empleo, legislación estatal y comunitaria, contratos...). Por último, el SEPE también nos ofrece un observatorio ocupacional del que se desprenden informes sobre el mercado laboral, tendencias, perfiles ocupacionales, estudios prospectivos sectoriales...etc.

b) Portal de empleo de la Xunta de Galicia. Web perteneciente al servicio público de empleo gallego y está destinada, fundamentalmente, a las personas desempleadas. Ofrece abundante información sobre ofertas de empleo, oferta de formación para personas desempleadas en Galicia, sobre la red EURES, y sobre servicios de orientación laboral destinados a personas desempleadas. A través de la oficina virtual permite hacer distintas gestiones (demanda de servicios, renovación y recuperación de la demanda, listas de ofertas de empleo, de cursos, adscripción a los mismos, modificación del CV...)

c) Red Eures. Web que proporciona información de empleo en Europa. Se encuentra distribuido en cuatro secciones: solicitantes de empleo, empresas, vida y trabajo y capacidades y desarrollo profesional.

A través del portal EURES podemos acceder a toda aquella información importante sobre movilidad laboral, funciones de búsqueda de empleo y a una red de casi 800 consejeros EURES a los que poder realizar cualquier consulta.

Dispone de información sobre la búsqueda de empleo en 31 países que se actualizan en tiempo real. A través del registro en la web podemos redactar nuestro $\mathrm{CV}$ y ponerlo a disposición de los empresarios ya registrados y de los consejeros EURES con el objetivo de acceder a las ofertas de empleo.

A través de ésta Red podemos acceder a multitud de información referente a todos los países de la Unión Europea (situación laboral y social, calidad de vida, aspectos jurídicos, administrativos...). A través de soportes de apoyo como Twitter, Facebook, LinkedIn y Google+ o su canal de YouTube, el usuario puede mantenerse al día y estar al tanto de cualquier información relevante.

d) Barcelona activa - Mercado. Web que nos ofrece información sobre 25 sectores económicos (tendencias, pautas de transformación, oportunidades profesionales). Además posee multitud de vídeos relacionados con cada una de los sectores así como informes referentes al mercado laboral en general así como a los sectores recogidos en particular.

\subsubsection{Información sobre autoempleo}

Una de las especializaciones que se le reconocen al profesor de FOL es la de asesoramiento en cuanto a las opciones de autoempleo, por lo que recogeremos, aquí algunas de las webs que proporcionan información útil en lo referente a esta temática. A continuación mostramos algunas de las webs que hemos seleccionado pertenecientes a esta temática: "Emprendedores y Pymes”(http://www.ipyme.org/es-ES/Paginas/Home.asp $\mathrm{x}$, "portal Circe" ( http://portal.circe.es/es-ES/Paginas/Home.aspx), “Todo emprende"(http://www.todoemprende.es/es-ES/Paginas/ home.aspx), cámara de comercio (http://www.camaracoruna.com/web/10169/1),

"Instituto gallego de promoción económica" 
(http://www.igape.es/es), “Confederación española de jóvenes empresarios (http://ceaje.es/).

\subsubsection{Información sobre empleo público}

Albergaremos aquí aquellas páginas web en las que se encuentre información interesante acerca de las diferentes posibilidades que ofrecen tanto organismos autonómicos y locales como estatales, como posible salida laboral. Algunas de las existentes en la red son: "Administración.gob.es" (http://administracion.gob.es/), "Secretaría de estado de administraciones Públicas" (http://www.seap.minhap.gob.es/servicios/empleo_publi co.html),Empleopúblico.net(http://www.empleopublico. net/),“Buscaoposiciones.com”(http://www.buscaoposici ones.com/), "Ministerio de asuntos exteriores y cooperación”http://www.exteriores.gob.es/Representaci onesPermanentes/EspanaUE/es/TrabajarenUE/Paginas/ TrabajarAdminUE.aspx, "Xunta de Galicia” (http://www.xunta.es/portada).

\section{Conclusiones}

La principal finalidad del catálogo, del que sólo presentamos una pequeña parte, ha sido, desde el principio, la de servir como herramienta de referencia para el profesorado del módulo de FOL a la hora de abordar la orientación profesional. Consideramos que es una aportación importante en la medida en que no existe un recurso de estas características, específicamente pensado para el profesorado de FOL. Su utilidad se deriva de su conexión con las funciones específicas de este profesional en el ámbito de la orientación profesional y del proceso de curación de contenidos realizado. Su importancia radica precisamente en la cantidad ingente de recursos existentes en la red y de la necesidad de su curación.

Las posibilidades que nos ofrece la web 2.0 en el ámbito educativo y concretamente en la orientación profesional son muy amplias por lo que deberemos aprovecharlas al máximo. La principal característica que se desprende de la web 2.0 en contraposición a la web 1.0 radica en su conectividad y dinamismo entre los diferentes usuarios de la red, por lo que la oportunidad que nos ofrece este avance tecnológico deberá de ser aprovechada por todos los profesionales de la docencia y concretamente, para nuestro caso, por el profesorado de FOL con la finalidad última de conseguir la mejora profesional y una mejor orientación de las personas, pues si se actúa conjuntamente, de manera colaborativa y nos separamos de ese aislamiento propio de la web 1.0 es muy probable que los resultados y la práctica docente aumenten su calidad. El conocimiento es de todos y por ello es positivo compartirlo con los demás, sobre todo cuando se trata de la orientación profesional, en el que, constantemente, tenemos que estar renovando nuestros conocimientos, pues éstos cambian a medida que lo hacen las nuevas tecnologías y por ello la conectividad a partir de la web 2.0 es tremendamente útil para los orientadores profesionales. En este sentido es fundamental que compartamos el espíritu social de la web 2.0 que se vincula al área de intervención de la orientación profesional. Por ello, este catálogo se pondrá a disposición de cualquier persona interesada a través de un marcador social, una de las herramientas que nos ofrece la web 2.0.

Al igual que la web 2.0 nos permite una conectividad entre profesionales de la orientación profesional sin precedentes, también en ella se alojan multitud de recursos, por lo que hoy más que nunca la curación de contenidos es cada vez más necesaria si queremos integrar las nuevas tecnologías en el aula. Este es uno de los cometidos del profesorado de FOL y como resultado se ha generado este catálogo de recursos que puede ser una herramienta interesante frente a la infoxicación a la que, en muchas ocasiones, profesionales y alumnos estamos sujetos. Tras revisar y analizar más de 100 recursos, finalmente, gracias al proceso de curación hemos conseguido filtrar algunos de los que creímos más útiles e interesantes en la red. A pesar de ello seguramente queden más recursos interesantes en la web, o que todavía se estén gestando, por lo que la actualización del catálogo servirá para incorporar dichos recursos en un futuro.

\section{Referencias}

Álvarez González, M. (1998). Modelos de intervención en orientación profesional. En Sobrado, L. (Ed.). Orientación e Inserción Profesional. Barcelona: Estel/ Universidade de Santiago de Compostela.

Álvarez González, M. e Isus, S. (1998). La orientación profesional. En Bisquerra, R. (Coord.) Modelos de orientación e intervención psicopedagógica. (233-259). Barcelona: Praxis.

Álvarez Gónzalez, M. (2009). Modelos explicativos de la Orientación Profesional. Universidad de Barcelona. En Sobrado, L. (2009). Orientación profesional. Nuevos escenarios y perspectivas. (47-72). Madrid: Biblioteca Nueva.

Álvarez González, M. \& Bisquerra Alzina, R. (2012). Orientación educativa. Modelos, áreas, estrategias y recursos. Madrid: Wolters Kluwer.

Arza, N. (2002). Aproximación conceptual a Orientación profesional. Orientación Profesional. Proxecto docente inédito. Universidade da Coruña.

Arza, N. y Reimundez, B. (2014). Catálogo de recursos de internet para orientación profesional. Comunicación presentada no XVI Congreso Internacional de Formación para o Traballo Norte de Portugal-Galiza. Instituto de Emprego e Formaçao Profissional, Porto, 16-17 de octubre de 2014.

Arza, N. (2015). Ámbitos de utilización da rede na orientación profesional. Documento inédito da materia de orientación e inserción laboral.

Baelo, R. (2013). Aplicaciones de las tecnologías de la información y la comunicación al ámbito dela orientación profesional. En Sánchez García, $M^{\circ}$ F. (2013) (coorda.) Orientación profesional y personal (pp.283-314). Madrid: UNED. 
Ceinos Sanz, C. (2009). Las tecnologías de la Información y comunicación (TIC) en Orientación Profesional. Universidad de Santiago de Compostela. En Sobrado, L. (2009). Orientación profesional. Nuevos escenarios y perspectivas. (183-201). Madrid: Biblioteca Nueva.

Fernandez Rey, E. (2009). La Orientación Académico-Profesional en los escenarios educativos. Universidad de Santiago. En Sobrado, L. (2009). Orientación profesional. Nuevos escenarios $y$ perspectivas. (203-220). Madrid: Biblioteca Nueva.

Romero, S (2002). Sugerencias para el desarrollo de la información y orientación profesional. Ponencia presentada al Seminario de la Sociedad Española de Pedagogía El Reto de la Formación Profesional. Madrid 6 y 7 de julio.
Sánchez García, M (2004). Orientación laboral. Para la diversidad y el cambio. Madrid: Sanz y Torres.

Sánchez García, M M F (2013) La intervención orientadora en las dimensiones profesional y personal En Sánchez García, M F. (2013). (coorda). Orientación profesional y personal (pp. 75-92). Madrid: UNED.

Sanjuán Roca, M $M^{\mathrm{a}}$ (2010). Los contenidos y las competencias del módulo de Formación y Orientación Laboral (FOL): Visión del profesorado en Galicia. Innovación educativa, $\mathrm{n}^{0}$ 20, 2010: pp. 89-103. Dpto. Didáctica y Organización Escolar Universidad de Santiago de Compostela. Recuperado de https://dspace.usc.es/bitstream/10347/5000/1/07.Sanj uan.pdf 